\title{
Laser Desorption/Ionization Fourier Transform Mass Spectrometry of Thin Films Deposited on Silicon by Plasma Polymerization of Acetylene
}

\author{
Saša M. Miladinović, ${ }^{\mathrm{a}}$ Valérie De Vriendt, ${ }^{\mathrm{b}}$ Scott A. Robotham, ${ }^{\mathrm{a}, \mathrm{c}}$ \\ Fabrizio Maseri, ${ }^{\text {d }}$ Stéphane Lucas, ${ }^{b}$ and Charles L. Wilkins ${ }^{a}$ \\ ${ }^{a}$ Chemistry and Biochemistry Department, University of Arkansas, Fayetteville, Arkansas, USA \\ ${ }^{b}$ PMR-LARN, University of Namur (FUNDP), Namur, Belgium \\ ${ }^{c}$ Nebraska Wesleyan University, Lincoln, Nebraska, USA \\ ${ }^{\mathrm{d}}$ ArcelorMittal Liège, Research and Development, Liège, Belgium
}

Thin films deposited on silicon substrate by three different methods of plasma polymerization of acetylene were analyzed by direct laser desorption/ionization Fourier transform mass spectrometry. High-resolution mass spectra showed the presence of carbon clusters and hydrocarbon oligomers in different relative abundances. During unipolar and continuous discharge polymerization of acetylene-hydrogen gas mixtures, quadrupole mass spectra of the plasma constituents showed the presence of molecular species with $\mathrm{m} / \mathrm{z}$ lower than 100 mainly peaks of $\mathrm{C}_{4} \mathrm{H}_{2}$ and $\mathrm{C}_{6} \mathrm{H}_{2}$. Films produced had smooth surfaces and the corresponding LDI-FTMS spectra displayed only carbon cluster signals in the positive ion mode and both hydrocarbon and carbon cluster signals (with much higher relative abundance of carbon cluster signals) in the negative ion mode. Alternatively, during bipolar discharge with either higher acetylene gas flux $\left(>40 \mathrm{~cm}^{3} / \mathrm{min}\right)$ or longer deposition times ( $\left.>10 \mathrm{~min}\right)$, quadrupole mass spectra of the plasma constituents showed signals corresponding to polycyclic aromatic hydrocarbons (PAH) with $\mathrm{m} / \mathrm{z}$ higher than 100. SEM pictures of the bipolar thin films demonstrated the presence of "flower" structures and nanoparticles developed on the surface. LDI-FTMS spectra of such thin films showed either total absence or lower relative abundance of carbon cluster signals, compared with hydrocarbon signals. (J Am Soc Mass Spectrom 2010, 21, 411-420) (c) 2010 American Society for Mass Spectrometry

$\mathrm{M}$ ass spectrometry (MS) is one of the most used analytical techniques for characterization of synthetic polymers [1-3]. Time-of-flight (TOF) mass analyzers are preferred for their analysis because of TOF's high sensitivity and the wide mass range of the resulting spectra [4]. However, the complexity of polymer samples is such that a mass analyzer with higher resolving power and high mass accuracy, such as a Fourier transform mass spectrometer, is advantageous [5]. Fourier transform mass spectrometry (FTMS), combined with tandem MS techniques, provides unambiguous oligomer determination, molecular weight distributions, and end group determinations [6-8].

Thin films produced by plasma polymerization of acetylene are used in many applications due to their extraordinary material properties, such as chemical inertness, infrared transparency and a low abrasion coefficient $[9,10]$. Plasma polymerization is a wellknown method for the dry deposition of thin polymeric

Address reprint requests to Dr. C. L. Wilkins, 251 Chemistry Bldg., University of Arkansas, Fayetteville, AR 72701, USA. E-mail: cwilkins@ uark.edu films [11, 12]. Compared with electrochemical or standard chemical processes, plasma polymerization has several advantages, such as good adhesion of polymer films to substrates and the ability to alter certain properties of the film by changing plasma parameters. It is also an environmentally friendly method because it is solvent-free and requires little energy. The films are usually prepared in low-temperature plasmas from a hydrocarbon precursor gas, which is dissociated and ionized in the discharge. Ions and radicals formed in the gas phase impinge on the substrates and lead to film growth [13].

Even though plasma polymerization has the ability to create thin smooth films on a wide variety of substrates, it can also generate nano- or micrometersized particles. In the microelectronics industry, the presence of nanoparticles in thin films is a problem, whereas in the catalytic and electromechanical industry, nanoparticles play a positive role due to their large surface to the volume ratio. Moreover, in the material science field, nanoparticles can be used to obtain a rough surface that increases the hydrophobic properties of film. Previous work showed that plas- 
mas, which generate nano- or micrometer-sized particles, can lead to the formation of novel interesting surfaces called nano- or microflowers [14]. To the best of our knowledge, this is the first time that such structure was observed for plasma polymerization of acetylene. Thus, for some applications, the presence of nanoparticles in thin films is required, whereas for other applications it is a major problem, and in the present research, smooth films are preferred. To be sure to obtain the right film for the right application, it is of fundamental importance to understand the formation and the growth mode of hydrocarbon polymer films (smooth or rough) obtained by plasma polymerization of acetylene. Therefore, detailed characterization of the films is necessary.

There are many possible reactions in the plasma phase during initiation of polymerization and also during film growth on the deposition substrate. Because of the limitations of common instruments for surface analysis, information about the plasma polymerization mechanism continues to be limited [15]. Surface analysis of such materials has mostly been conducted by X-ray photoelectron spectroscopy (XPS) and Raman spectroscopy [16-21]. Results of such analyses explain the elemental composition of the surface, electronic states of elements, binding energy, and offer molecular identification. However, due to the complexity of hydrocarbon plasma polymers, even high-resolution measurements with modern XPS and Raman instruments often produce ambiguous results. For determination of the composition of complex materials, secondary ion mass spectrometry (SIMS) is sometimes used. However, SIMS has some limitations that include the lack of intact molecular ion formation due to its tendency to produce primarily fragment ions $[22,23]$. The analytical techniques mentioned above are commonly used for film surface analysis. Usually, solid-state nuclear mag- netic resonance spectroscopy (SSNMR) is used to determine the bulk film structure. However, a major limitation of SSNMR is the number of samples that can be analyzed in a given period of time because for one sample, analysis time can be very long, up to a few days [24]. For the first time, we show that LDI-FTMS is a fast and appropriate technique to reveal the bulk structural characteristics of plasma polymers deposited on silicon substrate.

\section{Experimental}

\section{Film Production}

Thin films were synthesized by plasma polymerization of acetylene gas. The plasma was excited by three different methods: continuous (DC), unipolar pulsed (UP), and bipolar pulsed (BP) discharge. The discharge sequences are as follows. In pulsed discharges, the plasma is periodically switched "on" $\left(\mathrm{t}_{\mathrm{ON}}\right)$ then "off" $\left(t_{\mathrm{OFF}}\right)$. The discharge voltage is clamped close to ground potential in the "off" time. In general, the discharge voltage was around $480 \mathrm{~V}$. In producing the films a number of experimental parameters were varied, including the length of discharge applied $\left(t_{\mathrm{ON}}\right)$, the time between $t_{\mathrm{ON}}$ pulses $\left(\mathrm{t}_{\mathrm{OFF}}\right)$, the working pressure $(\mathrm{p})$ in vacuum chamber, the average discharge power $(\mathrm{P})$, and the acetylene gas flow $(\theta)$. Thin films were deposited on silicon substrates with the deposition parameters given in Table 1. The vacuum chamber for film deposition is described elsewhere [25]. A differentially pumped quadrupole mass spectrometer (Hiden PSM, Warrington, UK) was part of the deposition system for plasma polymerization and was used to analyze the plasma in the early stages of polymerization. The microand nanostructures of the resulting thin films were observed with a JEOL FEG-SEM microscope operating

Table 1. Plasma polymerization parameters of thin film samples

\begin{tabular}{|c|c|c|c|c|c|c|c|c|}
\hline $\begin{array}{l}\text { Sample } \\
\text { I.D. }\end{array}$ & $\begin{array}{l}\text { Discharge } \\
\text { method }\end{array}$ & $\begin{array}{l}\mathrm{t}_{\mathrm{ON}} \\
(\mu \mathrm{s})\end{array}$ & $\begin{array}{l}\mathrm{t}_{\mathrm{OFF}} \\
(\mu \mathrm{s})\end{array}$ & $\mathrm{p}$ (Torr) & $\mathrm{P}(\mathrm{W})$ & $\begin{array}{c}\text { Ö } \\
\left(\mathrm{cm}^{3} / \mathrm{min}\right)\end{array}$ & $\begin{array}{l}\text { Thickness } \\
\text { (nm) }\end{array}$ & $\begin{array}{c}\text { Surface } \\
\text { characteristic }\end{array}$ \\
\hline UP1 & Pulsed unipolar & 50 & 10 & $3.00 \mathrm{E}-03$ & 200 & 45 & 175 & Smooth \\
\hline UP2 & Pulsed unipolar & 50 & 30 & $3.00 \mathrm{E}-03$ & 200 & 45 & 124 & Smooth \\
\hline UP3 & Pulsed unipolar & 50 & 50 & $3.00 \mathrm{E}-03$ & 200 & 45 & 118 & Smooth \\
\hline UP4 & Pulsed unipolar & 5 & 90 & $5.00 \mathrm{E}-01$ & 10 & 45 & 10 & Rough \\
\hline UP5 & Pulsed unipolar & 5 & 90 & $3.00 \mathrm{E}-03$ & 10 & 45 & 62 & Smooth \\
\hline UP6 & Pulsed unipolar & 50 & 90 & 3.00E-03 & 10 & 45 & 86 & Smooth \\
\hline DC1 & Continuous & - & - & $3.00 \mathrm{E}-03$ & 200 & 45 & 302 & Smooth \\
\hline DC2 & Continuous & - & - & $3.00 \mathrm{E}-03$ & 10 & 45 & 115 & Smooth \\
\hline DC3 & Continuous & - & - & $5.00 \mathrm{E}-01$ & 10 & 45 & 10 & Rough \\
\hline BP1 & Pulsed bipolar & 5 & 90 & $5.00 \mathrm{E}-01$ & 40 & 11 & 130 & Smooth \\
\hline BP2 & Pulsed bipolar & 5 & 90 & $5.00 \mathrm{E}-01$ & 40 & 45 & 133 & Rough ("flowers") \\
\hline BP3 & Pulsed bipolar & 5 & 90 & $5.00 \mathrm{E}-01$ & 40 & 375 & 114 & $\begin{array}{l}\text { Rough ("flowers" + } \\
\text { nanoparticles) }\end{array}$ \\
\hline BP4 & Pulsed bipolar & 5 & 90 & $5.00 \mathrm{E}-01$ & 40 & 375 & 36 & Smooth \\
\hline BP5 & Pulsed bipolar & 5 & 90 & $5.00 \mathrm{E}-01$ & 40 & 375 & 84 & $\begin{array}{c}\text { Rough ("flowers" }+ \\
\text { nanoparticles) }\end{array}$ \\
\hline BP6 & Pulsed bipolar & 5 & 90 & $5.00 \mathrm{E}-01$ & 40 & 375 & 139 & $\begin{array}{c}\text { Rough ("flowers" }+ \\
\text { nanoparticles) }\end{array}$ \\
\hline
\end{tabular}


at $4.0 \mathrm{kV}$. The films' thickness and roughness were determined by a stylus profiler (Veeco Dektak, Dourdan, Cedex, France).

\section{Chemicals and Methods}

Aerodag G (a graphite dispersion in 2-propanol) was purchased from Ladd Research (Williston, VT, USA), anthracene was obtained from Fluka (Milwaukee, WI, USA), and dihydroxy benzoic acid (DHB) was purchased from Alfa Aesar (Ward Hill, MA, USA). LDIFTMS analysis of the graphite solution was done by directly spraying Aerodag $G$ onto either a stainless steel MALDI plate or a blank silicon chip. LDI-FTMS of anthracene was accomplished by adding $1 \mu \mathrm{L}$ of 0.5 $\mathrm{mM}$ anthracene solution in ethanol onto either the stainless steel MALDI plate or a blank silicon chip. For MALDI-FTMS analysis, the anthracene sample was mixed with DHB in molar ratio of 1:100, respectively. Methanol was used as a solvent.

LDI-FTMS experiments were performed using the external ionization source of a 9.4 Tesla IonSpec Ultima FTMS instrument (IonSpec/Varian Inc., Lake Forest, CA, USA). The spectrometer is equipped with $\mathrm{Nd}$ :YAG laser operated at a wavelength of $355 \mathrm{~nm}$ with an rf-only mode quadrupole, acting as an ion guide, connecting the ionization source to the analyzer region. The analyzer region was maintained at $\sim 10-11$ Torr, the ionization source at $\sim^{10-9}$ Torr, and nitrogen gas was used for ion cooling. Wide mass range spectra from $\mathrm{m} / \mathrm{z}$ 500-5000 were acquired using the gated trapping technique [26]. This mass range was chosen by obtaining LDI-TOF mass spectra (data not shown) of representative film samples produced by a different pulsed technique. A recent report of laser desorption mass spectrometry of polyacetylene showed much lower mass range spectra than those reported in the present study [27]. For FTMS spectra, a total of 6 desorption events followed by a single excitation was done per acquisition. One such acquisition was employed to generate each single spectrum. Thin film samples (1 $\mathrm{cm} 2$ ) were placed neat on a MALDI stainless steel target plate by affixing the sample with double-sided sticky tape.

The LDI-TOF spectra were qualitatively the same as the FTMS spectra over this mass range (except for the lower TOF resolution). MALDI-TOF experiments were accomplished using a Bruker Reflex III reflectron TOF spectrometer (Billerica, MA, USA) equipped with a 337-nm pulsed $\mathrm{N}_{2}$ laser (National Electronics, Inc, Miami, FL, USA).

\section{Results and Discussion}

Each film was analyzed in both positive and negative ion modes. The average molecular weights $\left(M_{n}\right.$ and $\mathrm{M}_{\mathrm{w}}$ ) and the polydispersity index (PD) for each sample were calculated from the spectral data using the following equations:

$$
M_{n}=\sum R_{i} M_{i} / \sum R_{i}
$$

$$
M_{w}=\sum R_{i} M_{i}^{2} / \sum R_{i} M_{i}
$$

$$
P D=M_{w} / M_{n}
$$

where the $M_{w}$ is the weight average molecular weight, $\mathbf{M}_{\mathrm{n}}$ is the number average molecular weight, $\mathbf{M}_{\mathbf{i}}$ is $m / z$ of the peak $i$, and $R_{i}$ is the corresponding relative abundance of peak $\mathrm{i}[6]$.

In the positive ion mode, LDI-FTMS spectra of unipolar pulsed discharge films (samples UP1, UP2, UP3, UP4, UP5, and UP6) carbon clusters are observed exclusively with peaks showing only even numbers of carbons separated by the mass difference of $C_{2}$. The lightest carbon cluster observed was $C_{50}$ and heaviest $\mathrm{C}_{360}$. All spectra measured had resolving power of $\sim 30,000$ and ppm error below 10. Figure 1a shows the positive ion mode LDI-FTMS spectrum of the UP1 sample. It can be seen that $\mathrm{C}_{60}$ and $\mathrm{C}_{70}$ clusters are notably more intense. This is also the case for all other UP samples. Because samples were analyzed without any pretreatment (addition of UV matrix or cationizing agent), the proposed ionization mechanism involves formation of radical cations $\left(\mathrm{M}^{+\bullet}\right)$ [28-30]. Unlike the results for the positive ion mode, the spectra observed in negative ion mode (Figure $1 \mathrm{~b}$ ) showed the presence of hydrocarbon oligomers in addition to carbon cluster signals. Negative ion mode FTMS spectra of UP films were observed with average resolving power of 40,000, enabling assignment of a number of isobaric peaks present in the spectrum. Errors of 10 ppm or better along with the isotopic patterns allowed mass assignments of oligomeric species covering the whole mass range with high certainty. The oligomer series consist of species containing carbon and hydrogen atoms with a large excess of carbon atoms. A series of oligomers are defined by the number of carbon atoms, where odd/ even numbers of carbon contain, respectively, odd/ even numbers of hydrogens. The series showed peaks differing in mass by the mass of $\mathrm{CH}$. Interestingly, $\mathrm{C}_{60}$ and $C_{70}$ clusters are also present in negative ion mode spectra but remarkably higher peak intensity than other carbon clusters was not observed, probably due to their lower stability as anions. Carbon clusters and hydrocarbons in this case appeared to form radical anions $\left(\mathrm{M}^{-\bullet}\right)$ upon laser desorption [28-30]. The absence of hydrocarbons in positive ion mode can be explained by the fact that laser desorption results in a high abundance of stable positive carbon cluster radical ions, while hydrocarbon ions are less stable due to the ease of hydrogen loss $[29,30]$. On the other hand, negative ion carbon clusters are less stable than cation carbon 

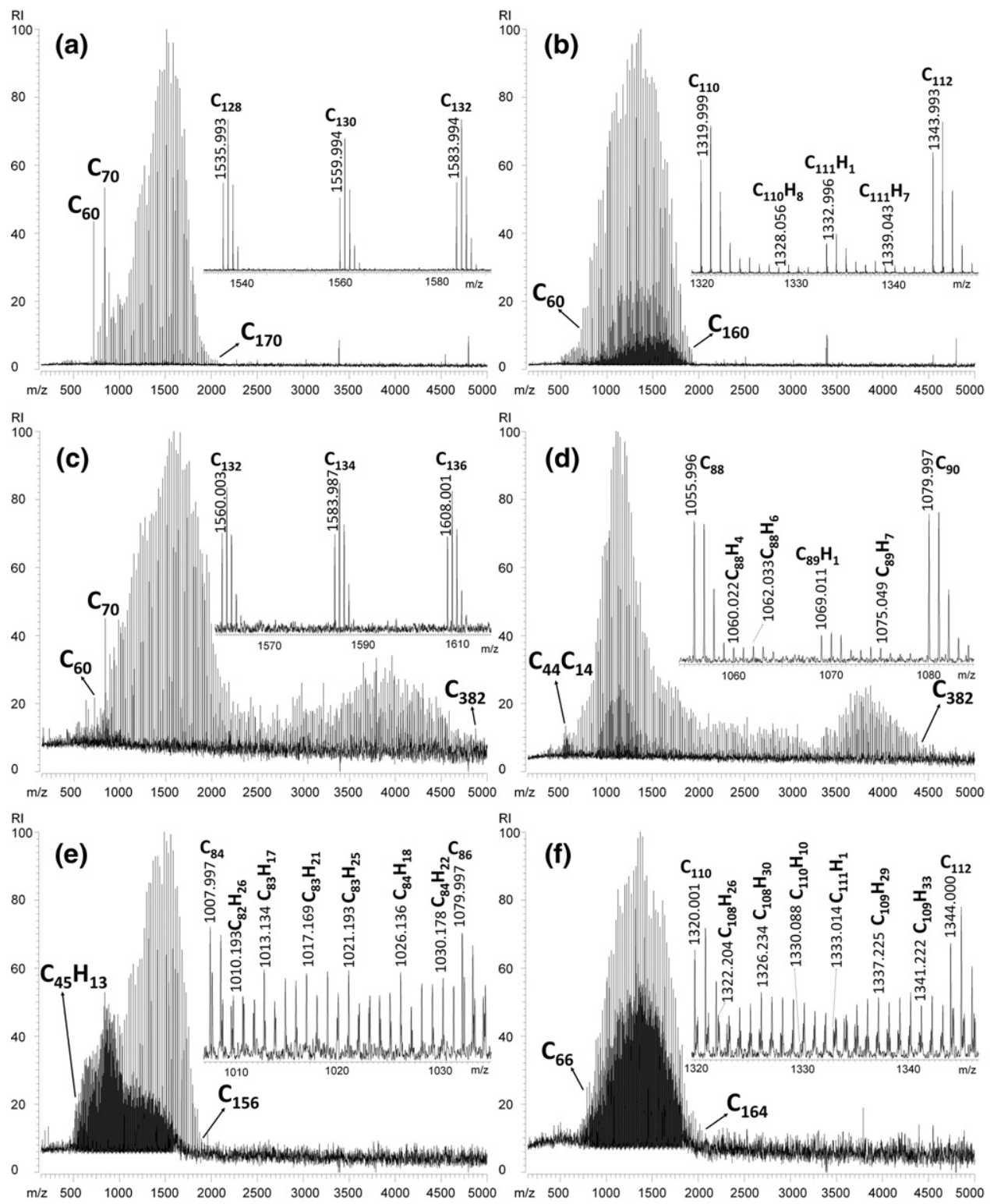

Figure 1. (a) Positive ion mode LDI-FTMS spectrum of UP1 sample, (b) negative ion mode LDI-FTMS spectrum of UP1 sample, (c) positive ion mode LDI-FTMS spectrum of DC1 sample, (d) negative ion mode LDI-FTMS spectrum of DC1 sample, (e) positive ion mode LDI-FTMS spectrum of BP1 sample, (f) negative ion mode LDI-FTMS spectrum of BP1 sample. Insets show a narrow $\mathrm{m} / \mathrm{z}$ region of each spectrum with selected peak assignments.

clusters, leading to the increased relative abundance of products derived from hydrocarbon negative radical ions $[29,31]$.

Only the UP4 thin film sample in this series of unipolar pulsed discharge films has a non-smooth surface. This physical characteristic influenced spot-to-spot reproducibility of the UP4 sample. Average molecular weights (Mw and $\mathrm{Mn}$ ) calculated from the two representative positive ion spectra acquired on two different rough spots of UP4 film showed differences of almost $1000 \mathrm{Da}$ (this average varied by as much as \pm 500 , Figure 2a and $b$ ), which was deemed to be significant. However, in both cases, only carbon clusters were observed with the different mass ranges. In both spectra, the lowest $\mathrm{m} / \mathrm{z}$ observable carbon cluster contained 50 carbon atoms, while the highest $\mathrm{m} / \mathrm{z}$ oligomer is composed of either 182 for low or 306 carbons for the higher $\mathrm{M}_{\mathrm{w}}$. When the location of the spot analyzed was changed on other UP thin films with smooth surfaces, spectra showed comparable molecular weights (differences less than $100 \pm 50$ ) and the same mass range. The molecular weights, polydispersity indices and plots of number of carbon atoms versus the number of hydrogen atoms in the oligomer series are summarized in Figure 3 (representative samples from three different pulse sequences) and Table S1 (supplementary mate- 


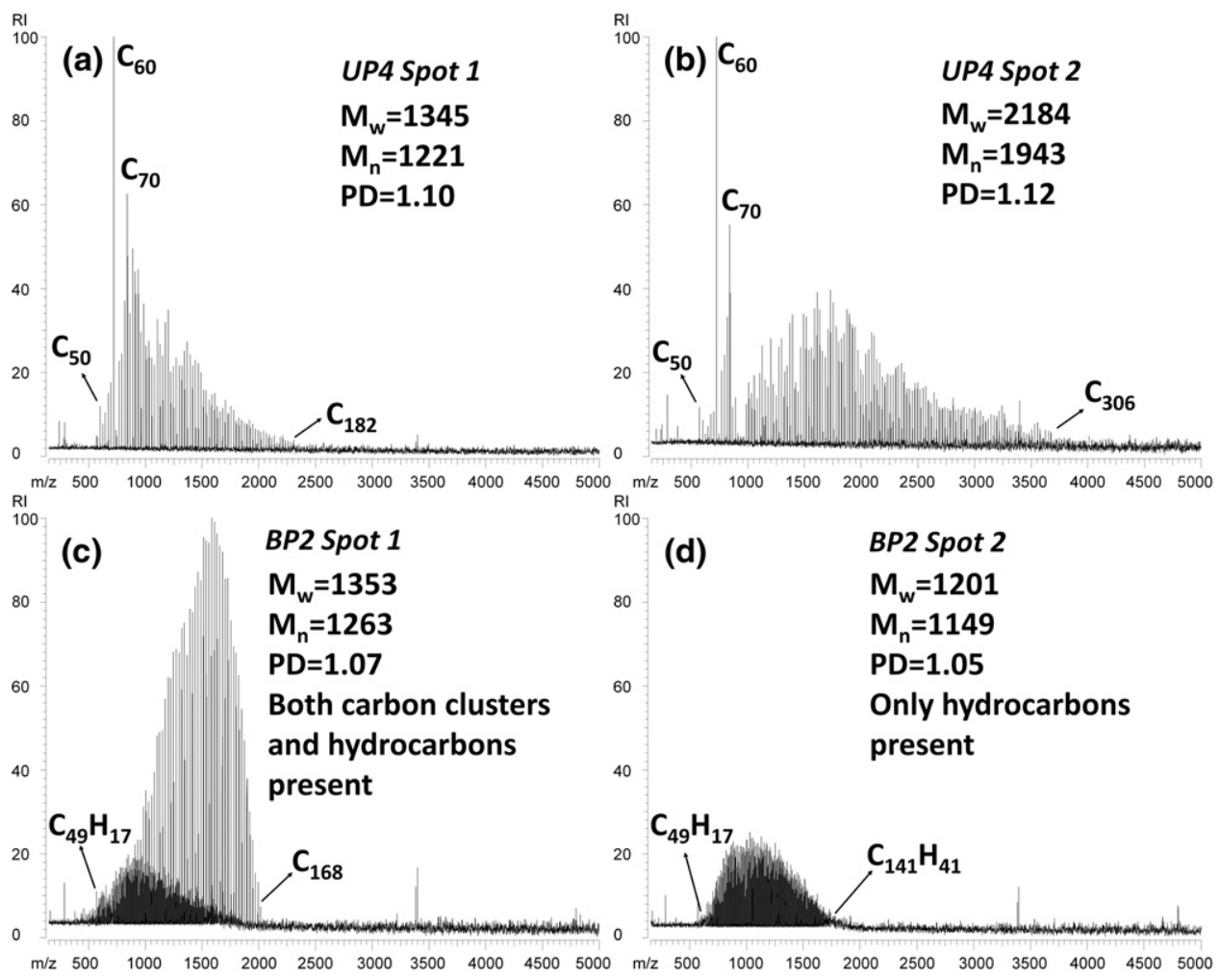

Figure 2. (a) and (b) Positive ion mode LDI-FTMS of sample UP4 that has slightly rough surface. Two spectra were recorded at two different spots on film's surface using identical laser power; (c) and (d) positive ion mode LDI-FTMS of sample BP2 that has rough surface with "flowers". Two spectra were recorded at two different spots on film's surface using identical laser power. Signal-to-noise ratio off all spectra were adjusted to the same value.

rial, data for all UP samples analyzed, which can be found in the electronic version of this article). Plots of positive mode LDI-FTMS are not shown since all spectra showed only carbon cluster signals.

For continuous discharge films (samples DC1, DC2, and DC3) taken in the positive ion mode again, only even numbered carbon cluster signals were observed with peaks separated by the mass difference corresponding to $\mathrm{C}_{2}$. The lightest carbon cluster observed was $C_{50}$ and heaviest was $C_{390}$. All spectra observed had resolving power of $\sim 30,000$ and ppm error below 10 . Figure 1c shows the positive ion mode LDI-FTMS spectrum of sample DC1 with a bimodal polymer distribution. The mass spectral properties of the DC1 sample in contrast to samples DC2 and DC3 showed this wider mass range bimodal distribution that may be explained by the thickness of the film. Most of the thin films were less than or about $100 \mathrm{~nm}$ thick, while DC1 was $302 \mathrm{~nm}$ thick (Table 1). Negative ion spectra of DC films showed the presence of hydrocarbon oligomers with abundant carbon cluster signals and bimodal polymer distributions (Figure 1d). The negative ion FTMS spectra of the DC films showed an average resolving power of 40,000 and mass measurement errors below $10 \mathrm{ppm}$. Similar to unipolar discharge films (the UP samples), the negative ion mass spectra consisted of carbon and hydrocarbon clusters with the hydrocarbons having both odd/even numbers of carbon atoms with lower numbers of either odd/even numbers of hydrogens. Essentially, in negative ion mode, the ratio between carbon cluster and hydrocarbon signal intensity did not differ from those of the UP films. Plots of the number of carbons versus the number of hydrogens have a shape similar to those seen for UP films (Figure 3 and Table S2, supplementary material). However, the plots of UP3, UP6, and DC1 showed interesting satellite features corresponding to the presence of groups of oligomers with either higher hydrogen (UP3 and DC1) or higher carbon content (UP6) compared with other film samples. No additional information can be deduced from those differences (Table S1 and Table S2, supplementary material).

The most interesting hydrocarbon plasma polymer samples were produced by the bipolar pulsed discharge method. SEM pictures of some BP films (Figure 4) showed the presence of spherical structures ("nanoparticles") and unique, particular structures called "flowers" [25]. It can be seen from Table 1 that some UP or DC thin films have rough surfaces but this roughness is not caused by "nanoparticles" or "flowers" formation. Figure 1e and $f$ show both positive and negative ion spectra of sample BP1. The resulting spectra have lower resolving power $(\sim 20,000)$ compared with UP and DC film spectra due to the lower signal intensities since 


\begin{tabular}{|c|c|c|c|}
\hline $\begin{array}{l}\text { Film } \\
\text { ID }\end{array}$ & $\begin{array}{l}\text { FTMS Ion } \\
\text { Mode }\end{array}$ & $\begin{array}{l}\text { Molecular } \\
\text { weight } \\
\text { calculated by } \\
\text { FTMS } \\
\end{array}$ & $\begin{array}{l}\text { Carbon versus hydrogen } \\
\text { plots, color scale represents } \\
\text { relative intensity of FTMS } \\
\text { signal }\end{array}$ \\
\hline \multirow[t]{2}{*}{$F$} & positive & $\begin{array}{l}M_{w}=1535 \\
M_{n}=1479 \\
P D=1.04\end{array}$ & Carbon cluster signals only \\
\hline & negative & $\begin{array}{l}M_{w}=1565 \\
M_{n}=1517 \\
P D=1.03\end{array}$ & 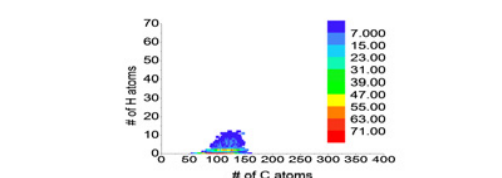 \\
\hline \multirow[t]{2}{*}{$\rightleftharpoons$} & positive & $\begin{array}{c}M_{w}=1660 \text { and } 3813 \\
M_{n}=1543 \text { and } 3753 \\
P D=1.08 \text { and } 1.01\end{array}$ & $\begin{array}{l}\text { Carbon cluster signals only } \\
\text { Bimodal distribution }\end{array}$ \\
\hline & negative & $\begin{array}{c}M_{w}=1530 \text { and } 3780 \\
M_{n}=1313 \text { and } 3745 \\
P D=1.16 \text { and } 1.02\end{array}$ & 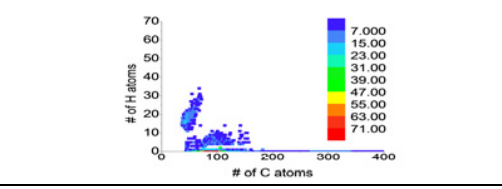 \\
\hline \multirow{2}{*}{ مि } & positive & $\begin{array}{l}\mathrm{M}_{\mathrm{w}}=1211 \\
\mathrm{M}_{\mathrm{n}}=1119 \\
\mathrm{PD}=1.08\end{array}$ & 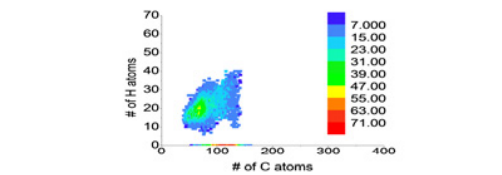 \\
\hline & negative & $\begin{array}{l}M_{w}=1407 \\
M_{n}=1354 \\
P D=1.04\end{array}$ & 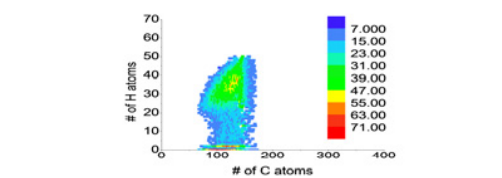 \\
\hline
\end{tabular}

Figure 3. Representative film data obtained by LDI-FTMS.

more oligomers were observed in BP spectra. Noticeably lower abundance carbon cluster signals were also observed. Moreover, hydrocarbon signals were present in both negative and positive ion mode spectra with much higher abundance than the carbon cluster signals previously described for UP and DC samples. This can be seen from Figure 3 (and Table S3, supplementary material) and plots of the number of carbons versus the number of hydrogens for representative spectra. In the plots for BP films it is observed that more intense peaks contain a greater number of hydrogens. Samples BP1, BP2, and BP3 were polymerized using the same parameters but varying only the acetylene gas flow. BP1 had the lowest gas flow $\left(11 \mathrm{~cm}^{3} / \mathrm{min}\right)$ producing smooth surfaces. The resulting BP1 LDI-FTMS spectra showed a higher abundance of carbon clusters than BP2 and BP3 samples. The BP2 film was produced using a higher gas flow $\left(45 \mathrm{~cm}^{3} / \mathrm{min}\right)$, which led to the formation of "flower" structures on the surface. However, there were few formed "flowers" and these were separated by smooth regions. This irregularity influenced spot-tospot reproducibility for positive ion LDI-FTMS spectra
(Figure 2c and d). Two types of spectra were observed from this sample, one producing carbon clusters and the other showing only the presence of hydrocarbons. Negative ion mode LDI-FTMS did not show any significant carbon cluster signals for this sample. For the BP3 thin film, an acetylene gas flow of $375 \mathrm{~cm}^{3} /$ min was used, leading to formation of numerous "flower" structures on the film's surface. Neither positive nor negative ion LDI-FTMS showed any significant carbon cluster signals. From this series of samples, it is obvious that there is a relation between the thin film's surface morphology and the observed LDI-FTMS spectra. When the "flowers" were present on thin film's surface, recorded spectra included only hydrocarbon oligomers. On the other hand, smooth surfaces produced carbon clusters peaks along with hydrocarbon signals.

A second series of $\mathrm{BP}$ thin films were formed varying the deposition time. BP4 was deposited for 5 min, BP5 for $10 \mathrm{~min}$, and BP6 for $12 \mathrm{~min}$. This influenced both the thickness and the roughness of films. Positive ion mode LDI-FTMS for BP4 (smooth surface) showed the presence of carbon clusters only, 

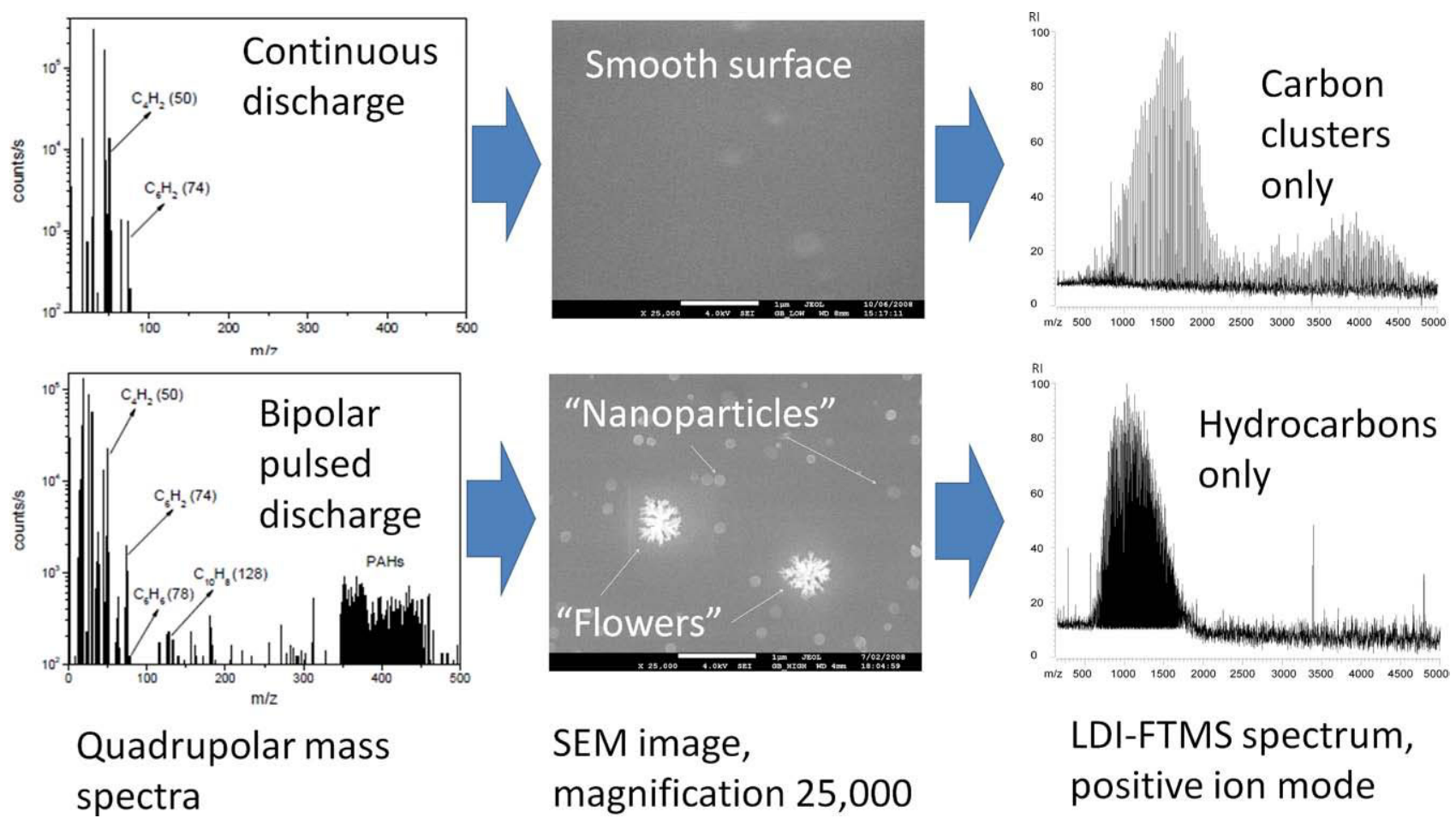

\section{LDI-FTMS spectrum, positive ion mode}

Figure 4. Relationship between quadrupole mass spectra of the plasma constituents, surface SEM image (scale: $1 \mu \mathrm{m}$ ), and positive ion mode LDI-FTMS for continuous discharge thin film (DC1, upper row) and bipolar pulsed thin film (BP3, bottom row).

while BP5 and BP6 ("flower" and "nanoparticle" formation, Table 1) showed the exclusive presence of hydrocarbons. Negative ion spectra for all samples from this series of thin films contained only hydrocarbon signals (Table S3, supplementary materia). The intensity of the observed signals increased with an increase in film thickness.

An interesting observation was made in the comparison of the quadrupole mass spectra of the plasma constituents, obtained during polymerization, and the LDI-FTMS mass spectra of the thin films produced (Figure 4). During polymerization at $3 \times 10^{-3}$ Torr with a continuous and unipolar pulsed discharge, quadrupole mass spectra of the plasma constituents showed the presence of molecular species with $\mathrm{m} / \mathrm{z}$ lower than 100 , mainly peaks of $\mathrm{C}_{4} \mathrm{H}_{2}$ and $\mathrm{C}_{6} \mathrm{H}_{2}$ (Figure 4, upper row). The films produced in these conditions had smooth surfaces and the corresponding LDI-FTMS spectra showed only carbon cluster signals for positive ion mode and both hydrocarbon and carbon cluster signals (with much higher relative intensity of carbon cluster signals) in negative ion mode. Alternatively, when bipolar pulsed discharge was employed at $5 \times$ $10^{-1}$ Torr with a high acetylene gas flow $\left(375 \mathrm{~cm}^{3} / \mathrm{min}\right)$ during a certain time (>10 min), the quadrupole mass spectra of the plasma constituents showed signals corresponding to polycyclic aromatic hydrocarbons (PAHs) with $\mathrm{m} / \mathrm{z}$ higher than 100 (Figure 4, bottom row) [25]. The films formed using these conditions had "nanoparticles" and "flowers" on their surface. LDI-FTMS spectra showed either a complete absence or lower relative abundance of carbon clusters compared with hydrocarbon signals. Therefore, when PAH molecules were present in the plasma, the films produced had nonsmooth surfaces and the resulting LDI-FTMS spectra contained abundant hydrocarbon signals (Figure 4). Interestingly, spot-to-spot reproducibility of the sample was worse when there were few flower structures on the film's surface and those structures were separated by regions of smooth surface which can be seen from SEM image. In one case, LDI-FTMS analysis produced spectra with only hydrocarbon signals (similar to nonsmooth thin film samples with "flowers") and in the other, both hydrocarbon and carbon cluster signals (similar to smooth thin film samples) were seen (Figure $2 \mathrm{c}$ and $\mathrm{d}$ ). Therefore, there is some reason to believe that flower structures and nanoparticles are made of $\mathrm{PAH}$ molecules, whereas the smooth regions contain carbon cluster structures.

A previous investigation on laser desorption ionization mass spectrometry of PAHs showed fragmentation of molecular ions in the observed spectrum [32]. Infrared LDI-FTMS of benzene soot (mixture of PAH) led to hydrogen loss in PAH molecules and formation of carbon clusters with the number of carbons exceeding 600 . When hexabenzocoronene and its derivatives were ionized by a UV laser in the absence of a matrix, molecular ions formed dimers and trimers with corresponding hydrogen loss. However, in the presence of a UV absorbing matrix, fragmentation and aggregation of 


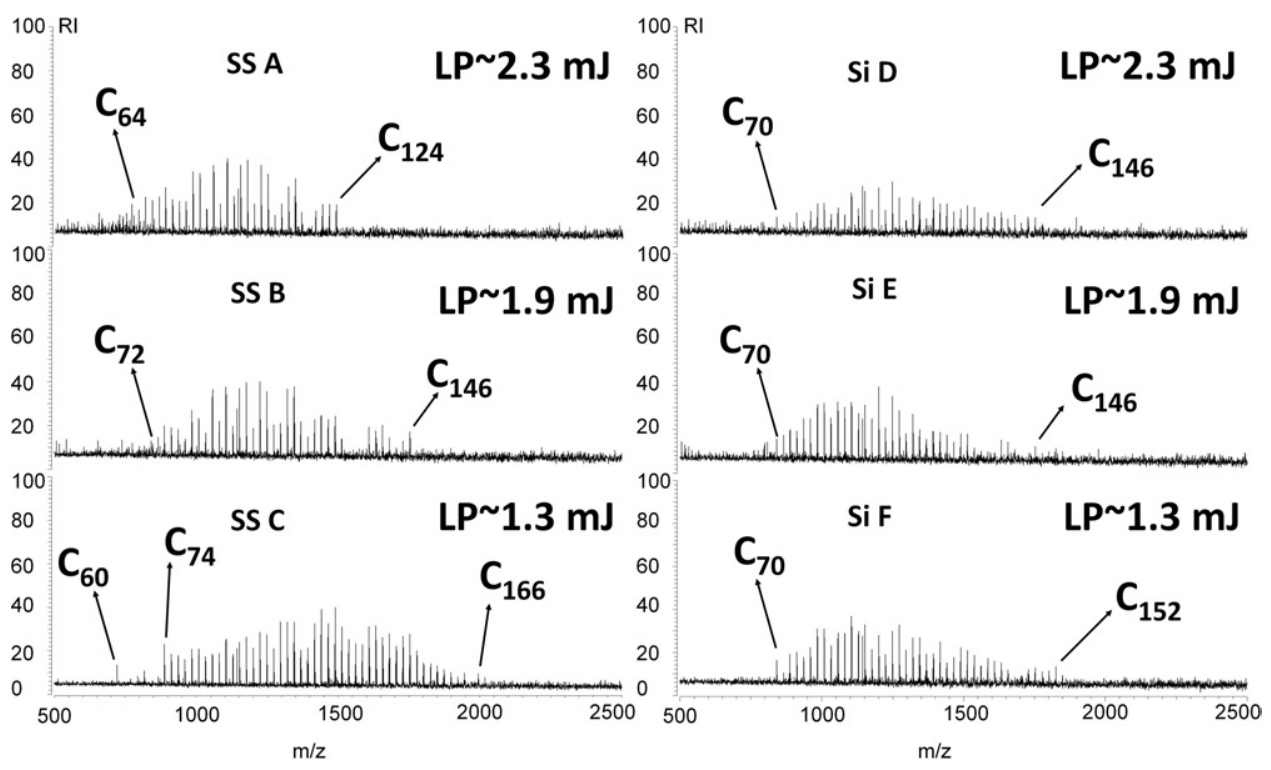

Figure 5. Positive ion mode LDI-FTMS of graphite (a) desorbed from stainless steel plate with laser power of $2.3 \mathrm{~mJ}$, (b) $1.9 \mathrm{~mJ}$, and (c) $1.3 \mathrm{~mJ}$; (d) desorbed from silicon with laser power of $2.3 \mathrm{~mJ}$, (e) 1.9 $\mathrm{mJ}$, and (f) $1.3 \mathrm{~mJ}$.

labile PAH molecules was reduced, and only molecular ions of PAH were observed without dimer and trimer formation [33]. Because the hydrocarbon plasma polymers were deposited on silicon, which serves as a UV matrix, carbon cluster formation is suppressed. An interesting investigation was conducted using laser ablation of a graphite target at $355 \mathrm{~nm}$ with a reflectron time-of-flight (TOF) mass spectrometer [34]. At low laser power, the mass spectra showed characteristic $\mathrm{C}_{n}{ }^{+}$ ions with $n=3-15$. With an increase in laser power, carbon cluster masses shifted to lower mass regions, indicating fragmentation of larger clusters within the hot plume. In this experiment, no additional UV matrix was used.

To examine possible fragmentation and aggregation, the same type of silicon plate as used to deposit hydrocarbon plasma polymer was employed to conduct LDI-FTMS experiments with graphite and anthracene (PAH) deposited on either a stainless steel plate or the blank silicon chips. Graphite was desorbed directly from the stainless steel plate at various the UV laser powers. It is noticeable from Figure $5 \mathrm{a}-\mathrm{c}$ that an increase in laser power shifted the mass distribution of carbon clusters to lower $\mathrm{m} / \mathrm{z}$. This is consistent with experiments performed by Koo et al. using a TOF mass analyzer [34]. On the other hand, when graphite was desorbed from silicon, the mass distribution of the observed spectra was constant (Figure $5 \mathrm{~d}-\mathrm{f}$ ), suggesting that fragmentation was suppressed when silicon is used as a sample support, which is also consistent with results reported previously [35, 36]. LDI-FTMS of anthracene was performed by direct laser desorption from both stainless steel and silicon surfaces, and by matrix assisted laser desorption/ionization (MALDI) using DHB as a matrix (Figure 6). The purpose of this exper- iment was to explore carbon cluster formation and possible fragmentations of PAHs with laser power slightly above the threshold. Experiments were performed using a different ion guide set-up. The First set-up covered the mass range from 200 to $400 \mathrm{Da}$. and the second from 400 to $1000 \mathrm{Da}$. When the lower mass range set-up was used anthracene desorbed from the silicon plate showed an abundant signal of a dimer with $\mathrm{H}_{2}$ loss and fragments of a dimer losing $\mathrm{CH}$ and $\mathrm{C}_{2} \mathrm{H}_{2}$. However, desorption performed from a stainless steel plate produced additional fragments of anthracene dimers with $\mathrm{C}_{3} \mathrm{H}_{3}$ loss together with an abundant peak of a protonated anthracene monomer-water cluster (Figure 6). Anthracene-water clusters and dimers were previously reported, $[37,38]$ although clusters with fewer molecules of water are not observed in our experiment. This is attributed to the low mass discrimination property of the ion guide. Interestingly, MALDI-FTMS over the $\mathrm{m} / \mathrm{z}$

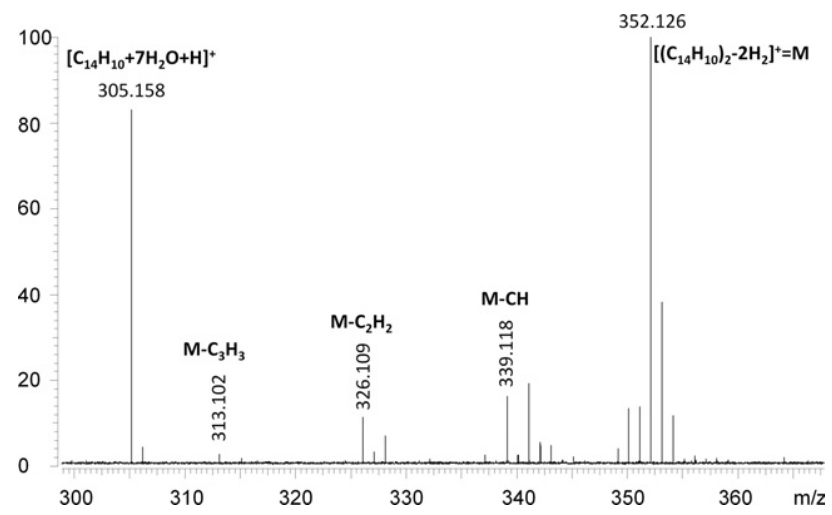

Figure 6. Positive ion mode LDI-FTMS of Anthracene desorbed from stainless steel. 
200-400 range showed only one peak corresponding to the protonated anthracene monomer-water cluster. The absence of such an ion during desorption from silicon plate can be explained by differences in the silicon and stainless steel surface morphology. The silicon plate is not porous while stainless steel has a large number of pores on its surface. Most likely, molecules of water from the moisture and solvent penetrated the stainless steel pores and during desorption/ionization were attached to the protonated anthracene ion.

When anthracene was desorbed from stainless steel with the ion guide covering the higher mass range $(\mathrm{m} / \mathrm{z}$ 400-1000), carbon cluster signals were observed with $\mathrm{C}_{60}$ as the most abundant signal. Carbon clusters were not present in the FTMS spectrum when anthracene was desorbed from silicon but the trimer was the most abundant ion with various $\mathrm{H}_{2}$ and $\mathrm{CH}$ losses (Figure 6). MALDI-FTMS did not produce any signal in this mass range. Clearly, when desorption of PAH is performed on silicon, hydrogen loss and fragmentation is limited compared with desorption from stainless steel.

All of the hydrocarbon plasma polymers were analyzed using various laser powers during the desorption/ ionization process. In both positive and negative ion spectra the mass distribution of observed spectra did not change with a change in laser power, showing similar behavior to that of graphite desorbed from a silicon chip. The exception was positive ion LDI-FTMS analysis of the UP1 sample where lower laser power produced spectra with a lower mass distribution of carbon clusters, while higher laser power produced a higher mass distribution which is opposite to the behavior of graphite desorbed from stainless steel. This occurrence was not understood completely since it was the only sample that showed this behavior.

\section{Conclusions}

Analysis of thin films deposited on silicon by plasma polymerization of acetylene using LDI-FTMS showed the presence of carbon clusters and hydrocarbon oligomers in different relative abundances. The bipolar pulsed plasma polymerization method formed relatively more hydrocarbon oligomers compared with those from unipolar pulsed and continuous discharge. The hydrocarbon oligomers were composed of carbon and hydrogen atoms with a large excess of carbon atoms. Broader mass range LDI-FTMS spectra are produced with increased film thickness. It can be hypothesized that if carbon clusters are produced from $\mathrm{PAH}$ (as a major component of a thin film sample) with lower laser power, some of the PAH signals would appear among the very intense carbon cluster signals. However, this was not the case for the analyzed thin films. This result, together with the limited fragmentation of graphite and anthracene on silicon, leads to the conclusion that carbon clusters are present in the thin film samples and are possibly formed during plasma polymerization.

\section{Acknowledgments}

Wilkins' group gratefully acknowledges support from NSF grants CHE 0455134 and CHE 0091868. Lucas' group acknowledges the "Fonds pour la Formation à la Recherche dans l'Industrie et l'Agriculture (FRIA)" and ArcelorMittal Research Liège in Belgium for support.

\section{Appendix A Supplementary Material}

Supplementary material associated with this article may be found in the online version at doi:10.1016/ j.jasms.2009.11.005.

\section{References}

1. Hanton, S. D. Mass Spectrometry of Polymers and Polymer Surfaces. Chem. Rev. 2001, 101, 527-569.

2. Peacock, P. M.; McEwen, C. N. Mass Spectrometry of Synthetic Polymers. Anal. Chem. 2006, 78, 3957-3964.

3. Weidner, S. M.; Trimpin, S. Mass Spectrometry of Synthetic Polymers. Anal. Chem. 2008, 80, 4349-4361.

4. Nielsen, M. W. F. MALDI Time-of-Flight Mass Spectrometry of Synthetic Polymers. Mass Spectrom. Rev. 1999, 18, 309-344.

5. Marshall, A. G.; Hendrickson, C. L.; Jackson, G. S. Fourier Transform Ion Cyclotron Resonance Mass Spectrometry: A Primer. Mass Spectrom. Rev. 1998, 17, 1-35.

6. Dey, M.; Castoro, J. A.; Wilkins, C. L. Determination of Molecular Weight Distributions of Polymers by MALDI-FTMS. Anal. Chem. 1995, 67, 1575-1579.

7. Miladinovic, S. M.; Robotham, S. A.; Wilkins, C. L. Wide Mass Range Trapping Using a 7-T Internal Source Matrix-Assisted Laser Desorption/ Ionization Fourier Transform Mass Spectrometer. Anal. Bioanal. Chem. 2008, 392, 585-594.

8. Simonsick, W. J. Jr.; Petkovska, V. I. Detailed Structural Elucidation of Polyesters and Acrylates Using Fourier Transform Mass Spectrometry. Anal. Bioanal. Chem. 2008, 392, 575-583.

9. Biederman, H.; Slavnska, D. Plasma Polymer Films and Their Future Prospects. Surf. Coat. Technol. 2000, 125, 371-376.

10. von Keudell, A. Formation of Polymer-Like Hydrocarbon Films from Radical Beams of Methyl and Atomic Hydrogen. Thin Solid Films 2002, 402, 1-37.

11. Friedrich, J.; Kühn, G.; Mix, R.; Unger, W. Formation of Plasma Polymer Layers with Functional Groups of Different Type and Density at Polymer Surfaces and Their Interaction with Al Atoms. Plasma Process. Polym. 2004, 1, 28-50.

12. Han, L. M.; Timmons, R. B.; Lee, W. W.; Chen, Y.; Hu, Z. Pulsed Plasma Polymerization of Pentafluorostyrene: Synthesis of Low Dielectric Constant Dilms. J. Appl. Phys. 1998, 84, 439-445.

13. Neyts, E.; Bogaerts, A.; van de Sanden, M. C. M. Reaction Mechanisms and Thin a-C:H Film Growth from Low Energy Hydrocarbon Radicals. J. Phys. Conf. Ser. 2007, 86, 012020.

14. De Vriendt, V.; Maseri, F.; Nonet, A.; Lucas, S. Study of Nanoparticles Formation in a Pulsed Magnetron Discharge in Acetylene. Plasma Process. Polym. 2009, in press.

15. De Bleecker, K.; Bogaerts, A.; Goedheer, W. Detailed Modeling of Hydrocarbon Nanoparticle Nucleation in Acetylene Discharges. Phys. Rev. E 2006, 73, 1-16.

16. Riedo, E.; Magnano, E.; Rubini, S.; Sancrotti, M.; Barborini, E.; Piseri, P.; Milani, P. Cluster-Assembled Carbon Films with Different Nanostructures: A Spectroscopic Study. Solid State Commun. 2000, 116, 287-292.

17. Swain, B. P. The Analysis of Carbon Bonding Environment in HWCVD Deposited a-SiC:H Films by XPS and Raman Spectroscopy. Surf. Coat. Tech. 2006, 201, 1589-1593.

18. Haruyama, Y.; Kitagawa, T.; Kanda, K.; Matsui, S.; Gejo, T.; Toyoda, N.; Yamada, I. X-Ray Photoelectron Spectroscopy Study of Diamond-Like Carbon Thin Films Formed by Ar Gas Cluster Ion Beam-Assisted Fullerene Deposition. Jpn. J. Appl. Phys. 2008, 47, 3380-3383.

19. Le Du, G.; Celini, N.; Bargaya, F.; Poncin-Epaillard, F. RF PlasmaPolymerization of Acetylene: Correlation Between Plasma Diagnostics and Deposit Characteristics. Surf. Coat. Tech. 2007, 201, 5821-5851.

20. Louh, S. P.; Wong, C. H.; Hon, M. H. Effects of Acetylene on Property of Plasma Ámorphous Carbon Films. Thin Solid Films 2006, 498, 235-239.

21. Stepanovic, O.; Berndt, J.; Winter, J. Influence of Hydrogen Atoms on the Growth of Carbon Based Nanoparticles and of Thin Films from Reactive Plasmas. Thin Solid Films 2008, 516, 6565-6569.

22. Huan, C. H. A.; Wee, A. T. S.; Gopalakrishnan, R.; Tan, K. L.; Kang, E. T. Neoh, K. G.; Liaw, D. J. Static SIMS of Conjugated Polymers: Films of the Substituted Polyacetylenes. Synthetic Met. 1993, 53, 193-203. 
23. Wee, A. T. S.; Huan, C. H. A.; Gopalakrishnan, R.; Tan, K. L.; Kang, E. T.; Neoh, K. G.; Shirakawa, H. Static SIMS of Polyacetylene: The Effect of Chain Unsaturation. Synthetic Met. 1991, 45, 227-234.

24. Nelson, B. N.; Schieber, L. J.; Barich, D. H.; Lubach, J. W.; Offerdahl, T. J.; Lewis, D. H.; Heinrich, J. P.; Munson, E. J. Multiple-Sample Probe for Solid-State NMR Studies of Pharmaceuticals. Solid State Nucl. Magn. Reson. 2006, 29, 204-213.

25. De Vriendt, V.; Maseri, F.; Nonet, A.; Lucas, S. Study of Nanoparticles Formation in a Pulsed Magnetron Discharge in Acetylene. Plasma Processes Polym. 2009, in press.

26. O'Connor, P. B.; Costello, C. E. Application of Multishot Acquisition in Fourier Transform Mass Spectrometry. Anal. Chem. 2000, 72, 5125-5130.

27. Brinckerhoff, W. B.; Coli, P.; Nguyen, M.-J.; Raulin, F.; Cornish, T. J.; Ecelberger, S. A. Analysis of Titan Thiolins by Laser Desorption Mass Spectrometry. In Lunar and Planetary Science XXXVIII, 2007.

28. McCarley, T. D.; McCarley, R. L.; Limbach, P. A. Electron-Transfer Ionization in Matrix-Assisted Laser Desorption/Ionization Mass Spectrometry. Anal. Chem. 1998, 70, 4376-4379.

29. Knochenmuss, R. Ion Formation Mechanisms in UV-MALDI. Analyst 2006, 131, 966-986.

30. Zenobi, R.; Knochenmuss, R. Ion Formation in MALDI Mass Spectrometry. Mass Spectrom. Rev. 1998, 17, 337-366.

31. Creasy, W. R.; Zimmerman, J. A.; Ruoff, R. S. Fullerene Molecular Weight Distributions in Graphite Soot Extractions Measured by Laser
Desorption Fourier Transform Mass Spectrometry. J. Phys. Chem. 1993, 97, 973-979.

32. So, H. Y.; Wilkins, C. L. First Observation of Carbon Aggregate Ion $>$ C600+ by Laser Desorption Fourier Transform Mass Spectrometry. J. Phys. Chem. 1989, 93, 1184-1187.

33. Cristadoro, A.; Rader, H. J.; Mullen, K. Clustering of Polycyclic Aromatic Hydrocarbons in Matrix-Assisted Laser Desorption/Ionization and Laser Desorption Mass Spectrometry. Rapid Commun. Mass Spectrom. 2007, 21, 2621-2628.

34. Koo, Y.-M.; Choi, Y.-K.; Lee, K. H.; Jung, K.-W. Mass Spectrometric Study of Carbon Cluster Formation in Laser Ablation of Graphite at 355 nm. Bull. Korean Chem. Soc. 2002, 23, 309-314.

35. Okuno, S.; Wada, Y.; Arakawa, R. Quantitative Analysis of Polypropyleneglycol Mixtures by Desorption/Ionization on Porous Silicon Mass Spectrometry. Int. J. Mass Spectrom. 2005, 241, 43-48.

36. Hsu, N.-Y.; Tseng, S. Y.; Wu, C.-Y.; Ren, C.-T.; Lee, Y.-C.; Wong, C.-H.; Chen, C.-H. Desorption iIonization of Biomolecules on Metals. Anal. Chem. 2008, 80, 5203-5210.

37. Schiedt, J.; Weinkauf, R. Photodetachment Photoelectron Spectroscopy of Mass Selected Anions: Anthracene and the aAnthracene- $\mathrm{H}_{2} \mathrm{O}$ Cluster. Chem. Phys. Lett. 1997, 266, 201-205.

38. Edwards, W. F.; Jin, L.; Thies, M. C. MALDI-TOF Mass Spectrometry: Obtaining Reliable Mass Spectra for Insoluble Carbonaceous Pitches. Carbon 2003, 41, 2761-2768. 